\title{
Constructing Banach Spaces of Vector-Valued Sequences with Special Properties
}

\author{
Geraldo Botelho \& Vinícius V. Fávaro
}

\begin{abstract}
We develop new techniques to construct infinitedimensional Banach (or quasi-Banach) spaces formed by vectorvalued sequences with special properties. We also investigate when such Banach/quasi-Banach spaces can be constructed with the maximum possible dimension. Numerous concrete applications of the results we prove are provided; the following example is illustrative: given a Banach space $X$ and $p>1$, defining $p_{n}:=p-\frac{1}{\log (\log (n+4))}$, we prove the existence of a Banach space of maximal dimension formed, up to the null vector, by $X$-valued $p$-summable sequences not belonging to the Nakano space of $X$-valued sequences determined by $\left(p_{n}\right)_{n=1}^{\infty}$.
\end{abstract}

\section{Introduction}

The title Linear subsets of nonlinear sets in topological vector spaces of the recent survey [6] is a precise description of the field to which this paper belongs. It has been a long time that mathematicians have been searching for some linear structure in nonlinear environments, but the paper [2] and the works of Gurariy and his collaborators (see, e.g. $[3 ; 14 ; 16 ; 17]$ ) made the subject fashionable, and the long list of recent references in [6] ensures the vitality of the area. In this paper we continue the research initiated in $[4 ; 8 ; 9]$ on the existence of infinite-dimensional closed subspaces of Banach or quasi-Banach sequence spaces formed by sequences with special properties. The state of the art is described in [6, Section 2.4.2], and very recent contributions can be found in [13; $15 ; 23]$.

Let us explain how our results push the results of [4;9] quite further. Given a Banach space $X$, in [9] the authors introduce a large class of Banach or quasiBanach spaces formed by $X$-valued sequences, called invariant sequences spaces (see Definition 2.1), which encompasses several classical sequences spaces as particular cases (see [9, Example 1.2]). Roughly speaking, the main results of [4;9] prove that, for every invariant sequence space $E$ of $X$-valued sequences and every subset $\Gamma$ of $(0, \infty]$, there exist a closed infinite-dimensional subspace of $E$ formed, up to the null vector, by sequences not belonging to $\bigcup_{q \in \Gamma} \ell_{q}(X)$

Received September 15, 2014. Revision received January 23, 2015.

The first author was supported by CNPq Grant 305958/2014-3 and Fapemig Grant PPM-00326-13.

The second author was supported by Fapemig Grants CEX-APQ-01409-12, PPM-00086-14 and CNPq Grants 482515/2013-9 and 307517/2014-4. 
and a closed infinite-dimensional subspace of $E$ formed, up to the null vector, by sequences not belonging to $c_{0}(X)$. In Section 2 of this paper, we consider the following much more general situation: given Banach spaces $X$ and $Y$, a map $f: X \longrightarrow Y$, a set $\Gamma \subseteq(0,+\infty]$, and an invariant sequence space $E$ of $X$-valued sequences, we investigate the existence of closed infinite-dimensional subspaces of $E$ formed, up to the origin, by sequences $\left(x_{j}\right)_{j=1}^{\infty} \in E$ such that either

$$
\begin{aligned}
& \left(f\left(x_{j}\right)\right)_{j=1}^{\infty} \notin \bigcup_{q \in \Gamma} \ell_{q}(Y) \text { or }\left(f\left(x_{j}\right)\right)_{j=1}^{\infty} \notin \bigcup_{q \in \Gamma} \ell_{q}^{w}(Y) \text { or } \\
& \left(f\left(x_{j}\right)\right)_{j=1}^{\infty} \notin c_{0}(Y) .
\end{aligned}
$$

As usual, $\ell_{p}(X)$ and $\ell_{p}^{w}(X)$ are the Banach spaces ( $p$-Banach spaces if $0<$ $p<1$ ) of $p$-summable and weakly $p$-summable $X$-valued sequences, respectively, and $c_{0}(X)$ is the Banach space of norm null $X$-valued sequences. Letting $f$ be the identity on $X$, the cases of sequences $\left(x_{j}\right)_{j=1}^{\infty} \in E$ such that $\left(f\left(x_{j}\right)\right)_{j=1}^{\infty} \notin \bigcup_{q \in \Gamma} \ell_{q}(Y)$ or $\left(f\left(x_{j}\right)\right)_{j=1}^{\infty} \notin c_{0}(Y)$ recover the situation investigated in [4; 9]. So, the results proved in Section 2 generalize the previous results in two directions: we consider $f$ belonging to a large class of functions (see Definition 2.3), and we consider spaces formed by sequences $\left(x_{j}\right)_{j=1}^{\infty} \in E$ such that $\left(f\left(x_{j}\right)\right)_{j=1}^{\infty}$ does not belong to $\bigcup_{q \in \Gamma} \ell_{q}^{w}(Y)$, a condition much more restrictive than not belonging to $\bigcup_{q \in \Gamma} \ell_{q}(Y)$.

In Section 3 we go even further by investigating when these Banach/quasiBanach spaces of $X$-valued sequences can be constructed with maximal dimension, that is, with the same dimension as that of the invariant sequence space $E$. Refining the argument used in Section 2, we show that, in some cases, this maximal dimension can be actually achieved.

In order to simplify the statements of the results, we use the following terminology, by now quite standard: a subset $A$ of a topological vector space $V$ is spaceable if $A \cup\{0\}$ contains a closed infinite-dimensional subspace $W$ of $V$. If such a subspace $W$ can be chosen such that $\operatorname{dim}(W)=\operatorname{dim}(V)$, then $A$ is said to be maximal spaceable.

It is worth mentioning that our results do not follow from the results of [19] because we prove the spaceability of sets that are not, in general, complements of vector subspaces (neither complements of countable unions of vector subspaces). Even in the case of complements of vector subspaces, for instance, when $f$ is a linear operator and $\Gamma$ is a singleton, our results encompass the nonlocally convex range $0<p<1$, whereas the results of [19], although quite general, are confined to the locally convex case. Moreover, the question of maximal spaceability is not treated in [19].

After proving the main result of each section (Theorems 2.5 and 3.2), we give some concrete applications. In Section 2 we consider situations in which the function $f$ is an (eventually discontinuous) linear operator or homogeneous polynomial. 
For example, we prove that for any two complete nonequivalent norms $\|\cdot\|_{1}$ and $\|\cdot\|_{2}$ on an infinite-dimensional space $X$ and $p>0$, there exists an infinitedimensional Banach/quasi-Banach space formed, up to the origin, by $X$-valued $\|\cdot\|_{1}$ - $p$-summable non- $\|\cdot\|_{2}$-weakly $p$-summable sequences (Corollary 2.15). Moreover, in Proposition 2.16 we show that even functions $f$ without any algebraic structure give rise to interesting applications of Theorem 2.5. In Section 3 we apply Theorem 3.2 to show that for certain sequences $\left(p_{j}\right)_{j=1}^{\infty}$ of positive numbers, there exists an infinite-dimensional Banach space of maximal dimension formed, up to the origin, by $p$-summable $X$-valued sequences not belonging to the vector-valued Nakano sequence space determined by $\left(p_{j}\right)_{j=1}^{\infty}$.

From now on all Banach and quasi-Banach spaces are considered over a fixed scalar field $\mathbb{K}$, which can be either $\mathbb{R}$ or $\mathbb{C}$.

\section{Spaceability}

The results of this section are proved within the general framework of invariant sequence spaces introduced in [9].

Definition 2.1. Let $X \neq\{0\}$ be a Banach space.

(a) Given $x \in X^{\mathbb{N}}$, by $x^{0}$ we mean the zerofree version of $x$, that is: if $x$ has only finitely many nonzero coordinates, then $x^{0}=0$; otherwise, $x^{0}=\left(x_{j}\right)_{j=1}^{\infty}$ where $x_{j}$ is the $j$ th nonzero coordinate of $x$.

(b) By an invariant sequence space over $X$ we mean an infinite-dimensional Banach or quasi-Banach space $E$ of $X$-valued sequences satisfying the following conditions:

(b1) For $x \in X^{\mathbb{N}}$ such that $x^{0} \neq 0, x \in E$ if and only if $x^{0} \in E$, and in this case, $\|x\|_{E} \leq K\left\|x^{0}\right\|_{E}$ for some constant $K$ depending only on $E$.

(b2) $\left\|x_{j}\right\|_{X} \leq\|x\|_{E}$ for every $x=\left(x_{j}\right)_{j=1}^{\infty} \in E$ and every $j \in \mathbb{N}$.

An invariant sequence space is an invariant sequence space over some Banach space $X$.

Several classical sequence spaces are invariant sequence spaces (see [9, Example 1.2]). For an invariant sequence space over $X$, spaceability of sets of the form $E-\bigcup_{q \in \Gamma} \ell_{q}(X)$, where $\Gamma \subseteq(0,+\infty]$, and $E-c_{0}(X)$ was studied in [9]. Here we take this study further by investigating the spaceability of the following sets:

Definition 2.2. Let $X$ and $Y$ be Banach spaces, $E$ be an invariant sequence space over $X, \Gamma \subseteq(0,+\infty]$, and $f: X \longrightarrow Y$ be a function. We define the following sets:

$$
\begin{aligned}
C(E, f, \Gamma) & =\left\{\left(x_{j}\right)_{j=1}^{\infty} \in E:\left(f\left(x_{j}\right)\right)_{j=1}^{\infty} \notin \bigcup_{q \in \Gamma} \ell_{q}(Y)\right\}, \\
C^{w}(E, f, \Gamma) & =\left\{\left(x_{j}\right)_{j=1}^{\infty} \in E:\left(f\left(x_{j}\right)\right)_{j=1}^{\infty} \notin \bigcup_{q \in \Gamma} \ell_{q}^{w}(Y)\right\}, \quad \text { and } \\
C(E, f, 0) & =\left\{\left(x_{j}\right)_{j=1}^{\infty} \in E:\left(f\left(x_{j}\right)\right)_{j=1}^{\infty} \notin c_{0}(Y)\right\} .
\end{aligned}
$$


The case studied in [9] is simply the case of $C(E, f, \Gamma)$ and $C(E, f, 0)$ with $f$ being the identity on $X$. Observe that the case of $C^{w}(E, f, \Gamma)$ is not treated in [9] at all. Whenever we say that either $C(E, f, \Gamma)$, or $C^{w}(E, f, \Gamma)$, or $C(E, f, 0)$ is spaceable, we mean that it is spaceable in $E$.

Different (but closely related) types of subhomogeneous functions have been considered in the literature (see, e.g., [10;12;20]). Here we use a class of functions that encompasses the subhomogeneous functions as particular cases (see Example 2.4). The applications of subhomogeneous functions $f$ usually require that $f(0)=0$, so we have incorporated this condition to the definition:

Definition 2.3. A map $f: X \longrightarrow Y$ between normed spaces is said to be:

(a) Noncontractive if $f(0)=0$ and for every scalar $\alpha \neq 0$, there is a constant $K(\alpha)>0$ such that

$$
\|f(\alpha x)\|_{Y} \geq K(\alpha) \cdot\|f(x)\|_{Y}
$$

for every $x \in X$.

(b) Strongly noncontractive if $f(0)=0$ and for every scalar $\alpha \neq 0$, there is a constant $K(\alpha)>0$ such that

$$
|\phi(f(\alpha x))| \geq K(\alpha) \cdot|\phi(f(x))|
$$

for all $x \in X$ and $\phi \in Y^{\prime}$.

By the Hahn-Banach theorem, strongly noncontractive functions are noncontractive.

EXAMPLE 2.4. Subhomogeneous functions are noncontractive because:

(a) A map $f: X \longrightarrow Y$ such that $f(0)=0$ and, for every scalar $\alpha \neq 0$, there is a constant $\lambda_{\alpha}>0$ such that

$$
\|f(\alpha x)\| \geq|\alpha|^{\lambda_{\alpha}} \cdot\|f(x)\| \quad \text { for every } x \in X
$$

is noncontractive,

(b) A map $f: X \longrightarrow Y$ such that $f(0)=0$ and, for every scalar $\alpha \neq 0$, there is a constant $\lambda_{\alpha}>0$ such that

$$
|\phi(f(\alpha x))| \geq|\alpha|^{\lambda_{\alpha}} \cdot|\phi(f(x))| \text { for all } \phi \in Y^{\prime} \text { and } x \in X
$$

is strongly noncontractive.

It is plain that bounded and unbounded linear operators are strongly noncontractive (hence noncontractive) with $K(\alpha)=|\alpha|$ for every $\alpha \neq 0$ and that homogeneous polynomials (continuous or not) are strongly contractive (hence contractive) with $K(\alpha)=|\alpha|^{n}$ for every $\alpha \neq 0$, where $n$ is the degree of homogeneity.

Now we can prove the main result of this section.

THEOREM 2.5. Let $X$ and $Y$ be Banach spaces, $E$ be an invariant sequence space over $X, f: X \longrightarrow Y$ be a function, and $\Gamma \subseteq(0,+\infty]$.

(a) If $f$ is noncontractive, then $C(E, f, \Gamma)$ and $C(E, f, 0)$ are either empty or spaceable. 
(b) If $f$ is strongly noncontractive, then $C^{w}(E, f, \Gamma)$ is either empty or spaceable.

Proof. (a) Let us fix a notation. For $\alpha=\left(\alpha_{n}\right)_{n=1}^{\infty} \in \mathbb{K}^{\mathbb{N}}$ and $w \in X$, we denote

$$
w \otimes \alpha=\alpha \otimes w:=\left(\alpha_{n} w\right)_{n=1}^{\infty} \in X^{\mathbb{N}}
$$

The proof begins following the idea of the proof of [9, Thm. 1.3]. Assume that $C(E, f, \Gamma)$ is nonempty and choose $x \in C(E, f, \Gamma)$. Since $E$ is an invariant sequence space, we have $x^{0} \in E$, and the condition $f(0)=0$ guarantees that $x^{0} \in C(E, f, \Gamma)$. Writing $x^{0}=\left(x_{j}\right)_{j=1}^{\infty}$, we have that $x_{j} \neq 0$ for every $j$. Split $\mathbb{N}$ into countably many infinite pairwise disjoint subsets $\left(\mathbb{N}_{i}\right)_{i=1}^{\infty}$. For every $i \in \mathbb{N}$, set $\mathbb{N}_{i}=\left\{i_{1}<i_{2}<\cdots\right\}$ and define

$$
y_{i}=\sum_{j=1}^{\infty} x_{j} \otimes e_{i_{j}} \in X^{\mathbb{N}} .
$$

Observe that $y_{i}^{0}=x^{0}$, so $0 \neq y_{i}^{0} \in E$; hence, $y_{i} \in E$ for every $i$ because $E$ is an invariant sequence space. For $q \in \Gamma, q<+\infty$, we have $\sum_{j=1}^{\infty}\left\|f\left(x_{j}\right)\right\|_{Y}^{q}=$ $+\infty$ because $x^{0} \in C(E, f, \Gamma)$. If $+\infty \in \Gamma$, then by the same reason we have $\sup _{i}\left\|f\left(x_{i}\right)\right\|_{Y}=+\infty$. It follows that each $y_{i} \in C(E, f, \Gamma)$. Let $K$ be the constant of condition 2.1(b1) and define $\tilde{s}=1$ if $E$ is a Banach space and $\tilde{s}=s$ if $E$ is an $s$-Banach space, $0<s<1$. For $\left(a_{i}\right)_{i=1}^{\infty} \in \ell_{\tilde{s}}$,

$$
\begin{aligned}
\sum_{i=1}^{\infty}\left\|a_{i} y_{i}\right\|_{E}^{\tilde{s}} & =\sum_{i=1}^{\infty}\left|a_{i}\right|^{\tilde{s}} \cdot\left\|y_{i}\right\|_{E}^{\tilde{s}} \leq K^{\tilde{s}} \cdot \sum_{i=1}^{\infty}\left|a_{i}\right|^{\tilde{s}} \cdot\left\|y_{i}^{0}\right\|_{E}^{\tilde{s}} \\
& =K^{\tilde{s}} \cdot\left\|x^{0}\right\|_{E}^{\tilde{s}} \cdot \sum_{i=1}^{\infty}\left|a_{i}\right|^{\tilde{s}} \\
& =K^{\tilde{s}} \cdot\left\|x^{0}\right\|_{E}^{\tilde{s}} \cdot\left\|\left(a_{i}\right)_{i=1}^{\infty}\right\|_{\tilde{s}}^{\tilde{s}}<+\infty .
\end{aligned}
$$

Thus, $\sum_{i=1}^{\infty}\left\|a_{i} y_{i}\right\|_{E}<+\infty$ if $E$ is a Banach space and $\sum_{i=1}^{\infty}\left\|a_{i} y_{i}\right\|_{E}^{S}<+\infty$ if $E$ is an $s$-Banach space, $0<s<1$. In both cases the series $\sum_{i=1}^{\infty} a_{i} y_{i}$ converges in $E$; hence, the operator

$$
T: \ell_{\tilde{s}} \longrightarrow E, \quad T\left(\left(a_{i}\right)_{i=1}^{\infty}\right)=\sum_{i=1}^{\infty} a_{i} y_{i},
$$

is well defined. It is easy to see that $T$ is linear and injective. Thus, $\overline{T\left(\ell_{\tilde{s}}\right)}$ is a closed infinite-dimensional subspace of $E$. We just have to show that if $z=$ $\left(z_{n}\right)_{n=1}^{\infty} \in \overline{T\left(\ell_{\tilde{s}}\right)}, z \neq 0$, then $\left(f\left(z_{n}\right)\right)_{n=1}^{\infty} \notin \bigcup_{q \in \Gamma} \ell_{q}(Y)$. Given such a $z$, there are sequences $\left(a_{i}^{(k)}\right)_{i=1}^{\infty} \in \ell_{\tilde{s}}, k \in \mathbb{N}$, such that $z=\lim _{k \rightarrow \infty} T\left(\left(a_{i}^{(k)}\right)_{i=1}^{\infty}\right)$ in $E$. Note that, for each $k \in \mathbb{N}$,

$$
T\left(\left(a_{i}^{(k)}\right)_{i=1}^{\infty}\right)=\sum_{i=1}^{\infty} a_{i}^{(k)} y_{i}=\sum_{i=1}^{\infty} a_{i}^{(k)} \cdot \sum_{j=1}^{\infty} x_{j} e_{i_{j}}=\sum_{i=1}^{\infty} \sum_{j=1}^{\infty} a_{i}^{(k)} x_{j} e_{i_{j}} .
$$


Fix $r \in \mathbb{N}$ such that $z_{r} \neq 0$. Since $\mathbb{N}=\bigcup_{j=1}^{\infty} \mathbb{N}_{j}$, there are (unique) $m, t \in \mathbb{N}$ such that $e_{m_{t}}=e_{r}$. Thus, for each $k \in \mathbb{N}$, the $r$ th coordinate of $T\left(\left(a_{i}^{(k)}\right)_{i=1}^{\infty}\right)$ is the vector $a_{m}^{(k)} x_{t}$. Condition 2.1(b2) ensures that convergence in $E$ implies coordinatewise convergence, so

$$
z_{r}=\lim _{k \rightarrow \infty} a_{m}^{(k)} x_{t}=\left(\lim _{k \rightarrow \infty} a_{m}^{(k)}\right) x_{t}
$$

It follows that $\alpha_{m}:=\lim _{k \rightarrow \infty} a_{m}^{(k)} \neq 0$. On the one hand, we have

$$
\alpha_{m} x_{j}=\left(\lim _{k \rightarrow \infty} a_{m}^{(k)}\right) x_{j}=\lim _{k \rightarrow \infty} a_{m}^{(k)} x_{j}
$$

for every $j \in \mathbb{N}$. On the other hand, for $j, k \in \mathbb{N}$, the $m_{j}$ th coordinate of $T\left(\left(a_{i}^{(k)}\right)_{i=1}^{\infty}\right)$ is $a_{m}^{(k)} x_{j}$. So, coordinatewise convergence gives $\lim _{k \rightarrow \infty} a_{m}^{(k)} x_{j}=$ $z_{m_{j}}$. It follows that $z_{m_{j}}=\alpha_{m} x_{j}$ for every $j \in \mathbb{N}$. Observe that $m$, which depends on $r$, is fixed, so the natural numbers $\left(m_{j}\right)_{j=1}^{\infty}$ are pairwise distinct (recall that $\left.\mathbb{N}_{m}=\left\{m_{1}<m_{2}<\cdots\right\}\right)$. Since $\sum_{j=1}^{\infty}\left\|f\left(x_{j}\right)\right\|_{Y}^{q}=+\infty$ for every $q \in \Gamma$, $q \neq+\infty$, by Definition 2.3(a) we have

$$
\begin{aligned}
\sum_{n=1}^{\infty}\left\|f\left(z_{n}\right)\right\|_{Y}^{q} & \geq \sum_{j=1}^{\infty}\left\|f\left(z_{m_{j}}\right)\right\|_{Y}^{q}=\sum_{j=1}^{\infty}\left\|f\left(\alpha_{m} x_{j}\right)\right\|_{Y}^{q} \\
& \geq\left[K\left(\alpha_{m}\right)\right]^{q} \cdot \sum_{j=1}^{\infty}\left\|f\left(x_{j}\right)\right\|_{Y}^{q}=+\infty
\end{aligned}
$$

for every $q$ in $\Gamma, q \neq+\infty$. Besides, if $+\infty \in \Gamma$, then we have $\left(f\left(x_{j}\right)\right)_{j=1}^{\infty} \notin$ $\ell_{\infty}(Y)$ because $x^{0} \in C(E, f, \Gamma)$; therefore,

$$
\begin{aligned}
\sup _{n}\left\|f\left(z_{n}\right)\right\|_{Y} & \geq \sup _{j}\left\|f\left(z_{m_{j}}\right)\right\|_{Y}=\sup _{j}\left\|f\left(\alpha_{m} x_{j}\right)\right\|_{Y} \\
& \geq K\left(\alpha_{m}\right) \cdot \sup _{j}\left\|f\left(x_{j}\right)\right\|_{Y}=+\infty .
\end{aligned}
$$

This shows that $\left(f\left(z_{n}\right)\right)_{n=1}^{\infty} \notin \bigcup_{q \in \Gamma} \ell_{q}(X)$ and completes the proof that $z \in$ $C(E, f, \Gamma)$.

Assume now that $C(E, f, 0)$ is nonempty and choose $x \in C(E, f, 0)$. As before, $x^{0} \in E$ because $E$ is an invariant sequence space, and $x^{0} \in C(E, f, 0)$ because $f(0)=0$ and $x^{0}=\left(x_{j}\right)_{j=1}^{\infty}$ with $x_{j} \neq 0$ for every $j$. Proceeding as before, we define the vectors $y_{i} \in E$ for $i \in \mathbb{N}$. We know that $f\left(x_{i}\right) \nrightarrow 0$ because $x^{0} \in C(E, f, 0)$, so each $y_{i} \in C(E, f, 0)$. Define the injective linear operator $T: \ell_{\tilde{s}} \longrightarrow E$ as before and note that all we have to prove is that if $z=\left(z_{n}\right)_{n=1}^{\infty} \in \overline{T\left(\ell_{\tilde{s}}\right)}, z \neq 0$, then $\left(f\left(z_{n}\right)\right)_{n=1}^{\infty} \notin c_{0}(Y)$. To do so, construct the subsequence $\left(z_{m_{j}}\right)_{j=1}^{\infty}$ as before and recall that $z_{m_{j}}=\alpha_{m} x_{j}$ for every $j$, where $\alpha_{m} \neq 0$. Since $\left\|f\left(x_{j}\right)\right\|_{Y} \nrightarrow 0$ because $x^{0} \in C(E, f, 0)$, we 
have

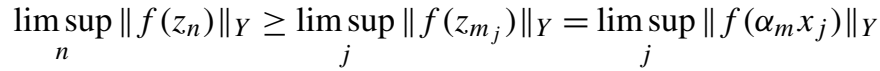

$$
\begin{aligned}
& \geq K\left(\alpha_{m}\right) \cdot \lim \sup _{j}\left\|f\left(x_{j}\right)\right\|_{Y}>0,
\end{aligned}
$$

which proves that $\left\|f\left(z_{n}\right)\right\|_{Y} \nrightarrow 0$, and hence $f\left(z_{n}\right) \nrightarrow 0$ in $Y$.

(b) If $+\infty \in \Gamma$, then there is nothing to prove because, in this case, $C^{w}(E, f, \Gamma)=C(E, f, \Gamma)$, which was treated in (a) with a weaker assumption on $f$. We thus can assume that $+\infty \notin \Gamma$. Assume that $C^{w}(E, f, \Gamma)$ is nonempty and choose $x \in C^{w}(E, f, \Gamma)$. Once again, $x^{0} \in E$ because $E$ is an invariant sequence space, and $x^{0} \in C^{w}(E, f, \Gamma)$ because $f(0)=0$ and $x^{0}=\left(x_{j}\right)_{j=1}^{\infty}$ with $x_{j} \neq 0$ for every $j$. Proceeding as before, we define the vectors $y_{i} \in E$ for $i \in \mathbb{N}$. For $q \in \Gamma$, since $x^{0} \in C^{w}(E, f, \Gamma)$, there is $\phi_{q} \in Y^{\prime}$ such that $\sum_{j=1}^{\infty}\left|\phi_{q}\left(f\left(x_{j}\right)\right)\right|^{q}=+\infty$, so each $y_{i} \in C^{w}(E, f, \Gamma)$. Define the injective linear operator $T: \ell_{\tilde{s}} \longrightarrow E$ as in the proof of (a) and note that all we have to prove is that if $z=\left(z_{n}\right)_{n=1}^{\infty} \in \overline{T\left(\ell_{\tilde{s}}\right)}, z \neq 0$, then $\left(f\left(z_{n}\right)\right)_{n=1}^{\infty} \notin \ell_{q}^{w}(Y)$ for every $q \in \Gamma$. To do so, construct the subsequence $\left(z_{m_{j}}\right)_{j=1}^{\infty}$ as before and recall that $z_{m_{j}}=\alpha_{m} x_{j}$ for every $j$, where $\alpha_{m} \neq 0$. Given $q \in \Gamma$, by Definition 2.3(b) we have

$$
\begin{aligned}
\sum_{n=1}^{\infty}\left|\phi_{q}\left(f\left(z_{n}\right)\right)\right|^{q} & \geq \sum_{j=1}^{\infty}\left|\phi_{q}\left(f\left(z_{m_{j}}\right)\right)\right|^{q}=\sum_{j=1}^{\infty}\left|\phi_{q}\left(f\left(\alpha_{m} x_{j}\right)\right)\right|^{q} \\
& \geq\left[K\left(\alpha_{m}\right)\right]^{q} \cdot \sum_{j=1}^{\infty}\left|\phi_{q}\left(f\left(x_{j}\right)\right)\right|^{q}=+\infty,
\end{aligned}
$$

proving that $z \notin \ell_{q}^{w}(Y)$.

Observe that [9, Thm. 1.3] is a particular case of Theorem 2.5(a) with $f$ being the identity operator on $X$. So, all consequences of [9, Thm. 1.3] listed in [9] are also consequences of Theorem 2.5. Next, we list some consequences of Theorem 2.5 that do not follow from [9, Thm. 1.3].

Definition 2.6. Let $X$ and $Y$ be Banach spaces, and $0<p \leq q<+\infty$. We say that

- a linear operator $u: X \longrightarrow Y$ is absolutely $(q, p)$-summing if $\left(u\left(x_{j}\right)\right)_{j=1}^{\infty} \in$ $\ell_{p}(Y)$ for each $\left(x_{j}\right)_{j=1}^{\infty} \in \ell_{q}^{w}(X)$.

- an $n$-homogeneous polynomial $P: X \longrightarrow Y$ is $p$-dominated if $\left(P\left(x_{j}\right)\right)_{j=1}^{\infty} \in$ $\ell_{p / n}(Y)$ for each $\left(x_{j}\right)_{j=1}^{\infty} \in \ell_{p}^{w}(X)$.

Corollary 2.7. Let $X$ and $Y$ be Banach spaces.

(a) Let $1 \leq p \leq q<+\infty$, and let $u: X \longrightarrow Y$ be a nonabsolutely $(q, p)$ summing linear operator. Then the set

$$
\left\{\left(x_{j}\right)_{j=1}^{\infty} \in \ell_{p}^{w}(X):\left(u\left(x_{j}\right)\right)_{j=1}^{\infty} \notin \ell_{q}(Y)\right\}
$$

is spaceable. 
(b) Let $0<p<+\infty$, and let $P: X \longrightarrow Y$ be a non- $p$-dominated $n$-homogeneous polynomial. Then the set

$$
\left\{\left(x_{j}\right)_{j=1}^{\infty} \in \ell_{p}^{w}(X):\left(P\left(x_{j}\right)\right)_{j=1}^{\infty} \notin \ell_{p / n}(Y)\right\}
$$

is spaceable.

Proof. Just apply Theorem 2.5 having in mind that linear operators and homogeneous polynomials are (strongly) noncontractive maps and that $\ell_{p}^{w}(X)$ is an invariant sequence space over $X$.

There are lots of continuous nonabsolutely $(q, p)$-summing linear operators studied in the literature. Many results asserting the existence of continuous non- $p$-dominated homogeneous polynomials are available (see [11] and references therein). They give us another opportunity to apply our results, for example:

Corollary 2.8. Let $X$ be an infinite-dimensional Banach space, $n \geq 3$, and $p \geq 1$. Then the set

$$
\left\{\left(x_{j}\right)_{j=1}^{\infty} \in \ell_{p}^{w}(X):\left(P\left(x_{j}\right)\right)_{j=1}^{\infty} \notin \ell_{p / n}\right\}
$$

is spaceable for some continuous scalar-valued $n$-homogeneous polynomial $P$ on $X$.

Proof. By [11, Thm. 2.4] there is a continuous scalar-valued non- $p$-dominated $n$-homogeneous polynomial on $X$. The result follows from Corollary 2.7(b).

We keep on using the standard terminology regarding linear operators and homogeneous polynomials:

Definition 2.9. Let $X$ and $Y$ be Banach spaces. The symbol $x_{j} \stackrel{w}{\longrightarrow} x$ means that the sequence $\left(x_{j}\right)_{j=1}^{\infty}$ is weakly convergent to $x$. We say that

- a linear operator $u: X \longrightarrow Y$ is completely continuous if $u\left(x_{j}\right) \longrightarrow u(x)$ in $Y$ whenever $x_{j} \stackrel{w}{\longrightarrow} x$ in $X$;

- an $n$-homogeneous polynomial $P: X \longrightarrow Y$ is weakly sequentially continuous at the origin if $P\left(x_{j}\right) \longrightarrow 0$ in $Y$ whenever $x_{j} \stackrel{w}{\longrightarrow} 0$ in $X$.

By $c_{0}^{w}(X)$ we denote the closed subspace of $\ell_{\infty}(X)$ formed by weakly null $X$ valued sequences. It is easy to check that $c_{0}^{w}(X)$ is an invariant sequence space over $X$, so from Theorem 2.5 we have the following:

Corollary 2.10. Let $X$ and $Y$ be Banach spaces.

(a) Let $u: X \longrightarrow Y$ be a noncompletely continuous linear operator. Then the set

$$
\left\{\left(x_{j}\right)_{j=1}^{\infty} \in c_{0}^{w}(X):\left(u\left(x_{j}\right)\right)_{j=1}^{\infty} \notin c_{0}(Y)\right\}
$$

is spaceable. In particular, if $X$ lacks the Schur property, then there exists an infinite-dimensional Banach space formed, up to the origin, by weakly null but not norm null $X$-valued sequences. 
(b) Let $P: X \longrightarrow Y$ be an n-homogeneous polynomial that fails to be weakly sequentially continuous at the origin. Then the set

$$
\left\{\left(x_{j}\right)_{j=1}^{\infty} \in c_{0}^{w}(X):\left(P\left(x_{j}\right)\right)_{j=1}^{\infty} \notin c_{0}(Y)\right\}
$$

is spaceable.

Again, linear operators that fail to be completely continuous and homogeneous polynomials that fail to be weakly sequentially continuous at the origin are easily found in the literature.

Our next purpose is to explore the possibility of considering discontinuous functions in Theorem 2.5. If $u: X \longrightarrow Y$ is an unbounded linear operator, then there is a sequence $\left(x_{j}\right)_{j=1}^{\infty}$ such that $x_{j} \longrightarrow 0$ in $X$ but $u\left(x_{j}\right) \nrightarrow 0$ in $Y$. We shall see, as a particular case of a more general result, that there is an infinitedimensional Banach space formed, up to the null vector, by sequences of this type.

Definition 2.11. A map $f: X \longrightarrow Y$ between Banach spaces is said to be:

- bounded if $f$ sends bounded subsets of $X$ to bounded subsets of $Y$,

- linearly strongly noncontractive near the origin if it is strongly noncontractive and there are $0<\varepsilon \leq 1$ and $t>0$ such that in Definition 2.3(b) we can choose $K(\alpha)=t|\alpha|$ whenever $0<|\alpha|<\varepsilon$.

Proposition 2.12. Let $X, Y$ be Banach spaces, and $f: X \longrightarrow Y$ be a map.

(a) If $f$ is discontinuous at the origin and noncontractive, then the set

$$
\left\{\left(x_{j}\right)_{j=1}^{\infty} \in c_{0}(X):\left(f\left(x_{j}\right)\right)_{j=1}^{\infty} \notin c_{0}(Y)\right\}
$$

is spaceable.

(b) If $f$ is unbounded and linearly strongly noncontractive near the origin, then the set

$$
\left\{\left(x_{j}\right)_{j=1}^{\infty} \in \ell_{p}(X):\left(f\left(x_{j}\right)\right)_{j=1}^{\infty} \notin \ell_{p}^{w}(Y)\right\}
$$

is spaceable for every $p>0$.

Proof. (a) The discontinuity of $f$ at the origin and the fact that $f(0)=0$ guarantee that $C\left(c_{0}(X), f, 0\right)$ is nonempty, so its spaceability follows from Theorem 2.5(a) because $c_{0}(X)$ is an invariant sequence space over $X$.

(b) Let us prove that $C^{w}\left(\ell_{p}(X), f,\{p\}\right) \neq \emptyset$ for $p>0$ : by the unboundedness of $f$ there is a bounded sequence $\left(x_{j}\right)_{j=1}^{\infty}$ in $X$ such that $\left(f\left(x_{j}\right)\right)_{j=1}^{\infty} \notin$ $\ell_{\infty}(Y)$. Since weakly bounded subsets of Banach spaces are norm bounded [21, Thm. 2.5.5], the set $\left\{f\left(x_{j}\right): j \in \mathbb{N}\right\}$ fails to be weakly bounded in $Y$. Thus, there is a functional $\phi \in Y^{\prime}$ such that $\left(\left|\phi\left(f\left(x_{j}\right)\right)\right|^{p}\right)_{j=1}^{\infty} \notin \ell_{\infty}$. By the duality $\ell_{1}^{\prime}=\ell_{\infty}$ and the Banach-Steinhaus theorem (uniform boundedness principle) there is a sequence $\left(\alpha_{j}\right)_{j=1}^{\infty} \in \ell_{1}$ such that the numerical series $\sum_{j=1}^{\infty} \alpha_{j}\left|\phi\left(f\left(x_{j}\right)\right)\right|^{p}$ diverges. In particular, $\sum_{j=1}^{\infty}\left|\alpha_{j}\right| \cdot\left|\phi\left(f\left(x_{j}\right)\right)\right|^{p}=+\infty$. Let $0<\varepsilon \leq 1$ and $t>0$ be 
as in the definition of linearly strongly noncontractive map at the origin, and let $N \in \mathbb{N}$ be such that $\left|\alpha_{j}\right|^{1 / p}<\varepsilon$ for every $j \geq N$. So $K\left(\left|\alpha_{j}\right|^{1 / p}\right)=t\left|\alpha_{j}\right|^{1 / p}$ for $j \geq N$, and hence

$$
\begin{aligned}
\sum_{j=N}^{\infty}\left|\phi\left(f\left(\left|\alpha_{j}\right|^{1 / p} x_{j}\right)\right)\right|^{p} & \geq \sum_{j=N}^{\infty}\left[K\left(\left|\alpha_{j}\right|^{1 / p}\right)\right]^{p} \cdot\left|\phi\left(f\left(x_{j}\right)\right)\right|^{p} \\
& =t^{p} \cdot \sum_{j=N}^{\infty}\left|\alpha_{j}\right| \cdot\left|\phi\left(f\left(x_{j}\right)\right)\right|^{p}=+\infty .
\end{aligned}
$$

Putting $z_{j}=\left|\alpha_{j}\right|^{1 / p} x_{j}$, we have that $\left(z_{j}\right)_{j=1}^{\infty} \in \ell_{p}(X)$ because $\left(x_{j}\right)_{j=1}^{\infty}$ is bounded and $\left(\alpha_{j}\right)_{j=1}^{\infty} \in \ell_{1}$, and, by the computation before, $\left(f\left(z_{j}\right)\right)_{j=1}^{\infty} \notin \ell_{p}^{w}(Y)$. It follows that $C^{w}\left(\ell_{p}(X), f,\{p\}\right) \neq \emptyset$; hence, it is spaceable by Theorem 2.5(b) because $\ell_{p}(X)$ is an invariant sequence space over $X$ for every $p>0$.

Recall that a subset $A$ of a topological vector space $E$ is maximal dense-lineable if $A \cup\{0\}$ contains a dense linear subspace $V$ of $E$ with $\operatorname{dim}(V)=\operatorname{dim}(E)$.

Proposition 2.13. Let $X$ and $Y$ be Banach spaces, and $p>0$. Then the sets

$$
\begin{aligned}
& \left\{\left(x_{j}\right)_{j=1}^{\infty} \in c_{0}(X):\left(u\left(x_{j}\right)\right)_{j=1}^{\infty} \notin c_{0}(Y)\right\} \quad \text { and } \\
& \left\{\left(x_{j}\right)_{j=1}^{\infty} \in \ell_{p}(X):\left(u\left(x_{j}\right)\right)_{j=1}^{\infty} \notin \ell_{p}^{w}(Y)\right\}
\end{aligned}
$$

are spaceable for every unbounded linear operator $u: X \longrightarrow Y$. Moreover, if $X$ is separable and $p<+\infty$, then these subsets are also maximal denselineable.

Proof. The first assertion follows from Proposition 2.12. We shall apply [5, Thm. 2.3(b)] to prove the second assertion. Assume that $X$ is separable and $p<+\infty$. It is easy to see that $c_{0}(X)$ and $\ell_{p}(X)$ are separable as well. Let $A$ be either $C\left(c_{0}(X), u, 0\right)$ or $C^{w}\left(\ell_{p}(X), u,\{p\}\right)$. By the first assertion we know that $A \cup\{0\}$ contains a $\mathfrak{c}$-dimensional subspace, where $\mathfrak{c}$ is the cardinality of the continuum. Let $c_{00}(X)$ denote the space of eventually null $X$-valued sequences. It is clear that $A+c_{00}(X) \subseteq A, A \cap c_{00}(X)=\emptyset$ and $c_{00}(X)$ is a dense infinitedimensional subspace of $c_{0}(X)$ and $\ell_{p}(X)$. By [5, Thm. 2.3(b)] $A \cup\{0\}$ contains a $\mathfrak{c}$-dimensional dense subspace, and the result follows because $c_{0}(X)$ and $\ell_{p}(X)$ are $\mathfrak{c}$-dimensional (recall that they are separable infinite-dimensional Banach or quasi-Banach spaces).

Example 2.14. Let $X$ be an infinite-dimensional Banach space, and $0<p<$ $+\infty$. In [9, Cor. 1.5] it is proved that $\ell_{p}^{w}(X)-\ell_{p}(X)$ is spaceable, that is, there exists an infinite-dimensional Banach/quasi-Banach space formed, up to the origin, by $X$-valued sequences $\left(x_{j}\right)_{j=1}^{\infty}$ such that $\sum_{j=1}^{\infty}\left|\phi\left(x_{j}\right)\right|^{p}<+\infty$ for every bounded linear functional $\phi \in X^{\prime}$ and $\sum_{j=1}^{\infty}\left\|x_{j}\right\|^{p}=+\infty$. Considering an unbounded linear functional $\phi$ on $X$, which always exists, Proposition 2.13 
yields the following dual result: there exists an infinite-dimensional Banach/quasiBanach space formed, up to the origin, by $X$-valued sequences $\left(x_{j}\right)_{j=1}^{\infty}$ such that $\sum_{j=1}^{\infty}\left\|x_{j}\right\|^{p}<+\infty$ and $\sum_{j=1}^{\infty}\left|\phi\left(x_{j}\right)\right|^{p}=+\infty$.

For the full appreciation of the next application, keep in mind that every infinitedimensional Banach space admits lots of complete nonequivalent norms (see, e.g., [1]). Given two complete nonequivalent norms $\|\cdot\|_{1}$ and $\|\cdot\|_{2}$ on $X$, it is expected that the sets $\ell_{p}\left(X,\|\cdot\|_{1}\right)-\ell_{p}\left(X,\|\cdot\|_{2}\right)$ and $\ell_{p}^{w}\left(X,\|\cdot\|_{1}\right)-\ell_{p}^{w}\left(X,\|\cdot\|_{2}\right)$ are spaceable (we are using the notation $A-B=A \cap B^{\complement}$ ). Applying Proposition 2.13 (and the open mapping theorem) we have much more:

Corollary 2.15. Let $\|\cdot\|_{1}$ and $\|\cdot\|_{2}$ be any two complete nonequivalent norms on an infinite-dimensional space $X$, and $p>0$. Then the sets

$$
c_{0}\left(X,\|\cdot\|_{1}\right)-c_{0}\left(X,\|\cdot\|_{2}\right) \quad \text { and } \quad \ell_{p}\left(X,\|\cdot\|_{1}\right)-\ell_{p}^{w}\left(X,\|\cdot\|_{2}\right)
$$

are spaceable. Moreover, if $X$ is separable and $p<+\infty$, then these subsets are also maximal dense-lineable.

Of course, the corollary can be adapted, mutatis mutandis, to any pair of nonisomorphic infinite-dimensional Banach spaces of the same dimension, for example, for any two nonisomorphic separable infinite-dimensional Banach spaces.

Applications of Theorem 2.5 go beyond linear operators and homogeneous polynomials. We give below an illustrative example. For the definition of $r$ regular maps, see [20, Def. 2.2]; examples, including and beyond linear operators and homogeneous polynomials, can be found in [20, Examples 2.4].

Proposition 2.16. Let $p, q>0, X, Y$ be Banach spaces, and $f: X \longrightarrow Y$ be a noncontractive map that fails to be $\frac{p}{q}$-regular at the origin. Then the set

$$
\left\{\left(x_{j}\right)_{j=1}^{\infty} \in \ell_{p}(X):\left(f\left(x_{j}\right)\right)_{j=1}^{\infty} \notin \ell_{q}(Y)\right\}
$$

is spaceable.

Proof. By [20, Thm. 2.5], the non- $\frac{p}{q}$-regularity of $f$ at the origin ensures that the set $C\left(\ell_{p}(X), f,\{q\}\right)$ is nonvoid, so its spaceability follows from Theorem 2.5(a).

\section{Maximal Spaceability}

Whereas in Section 2 we proved spaceability of certain sets of vector-valued sequences, in this section we move forward to prove maximal spaceability. With such a much more general conclusion, we are supposed to work with more restrictive conditions. To give our result more generality, we work with sequences of functions instead of one single function. In doing so, we give an interesting application (Corollary 3.3) of the main result of this section (Theorem 3.2) within the setting of classical Nakano sequence spaces. 
DEFinition 3.1. A sequence of functions $\left(f_{j}: \mathbb{K} \longrightarrow \mathbb{K}\right)_{j=1}^{\infty}$ is said to be:

(a) Noncontractive if $f_{j}(0)=0$ for every $j$ and for every scalar $\alpha \neq 0$, there is a constant $K(\alpha)>0$ such that

$$
\left|f_{j}(\alpha \xi)\right| \geq K(\alpha) \cdot\left|f_{j}(\xi)\right| \quad \text { for all } \xi \in \mathbb{K} \text { and } j \in \mathbb{N}
$$

and if $\inf \{K(\alpha):|\alpha|=1\}>0$.

(b) Nonincreasing near the origin if there are $C>0$ and $\varepsilon>0$ such that $\left|f_{n}(\xi)\right| \geq$ $C\left|f_{m}(\xi)\right|$ whenever $n \leq m$ and $|\xi|<\varepsilon$.

For $p>0$ and $\Gamma \subseteq(0,+\infty]$, define

$$
C\left(\ell_{p},\left(f_{j}\right)_{j=1}^{\infty}, \Gamma\right)=\left\{\left(\xi_{j}\right)_{j=1}^{\infty} \in \ell_{p}:\left(f_{j}\left(\xi_{j}\right)\right)_{j=1}^{\infty} \notin \bigcup_{q \in \Gamma} \ell_{q}\right\}
$$

THEOREM 3.2. Let $\left(f_{j}: \mathbb{K} \longrightarrow \mathbb{K}\right)_{j=1}^{\infty}$ be a noncontractive and nonincreasing near the origin sequence, $p>0$, and $\Gamma \subseteq(0,+\infty]$. If $C\left(\ell_{p},\left(f_{j}\right)_{j=1}^{\infty}, \Gamma\right)$ is nonempty, then

$$
C\left(\ell_{p}(X),\left(f_{j} \circ\|\cdot\|\right)_{j=1}^{\infty}, \Gamma\right):=\left\{\left(x_{j}\right)_{j=1}^{\infty} \in \ell_{p}(X):\left(f_{j}\left(\left\|x_{j}\right\|\right)\right)_{j=1}^{\infty} \notin \bigcup_{q \in \Gamma} \ell_{q}\right\}
$$

is maximal spaceable, regardless of the infinite-dimensional Banach space $X$.

Proof. The structure of the proof is similar to that of Theorem 2.5, but the differences are substantial, and it is worth giving a detailed argument.

By assumption there exists a scalar sequence $\xi=\left(\xi_{j}\right)_{j=1}^{\infty} \in \ell_{p}$ such that $\left(f_{j}\left(\xi_{j}\right)\right)_{j=1}^{\infty} \notin \bigcup_{q \in \Gamma} \ell_{q}$. Since $f_{j}(0)=0$ for every $j$, it is clear that we may suppose that $\xi_{j} \neq 0$ for every $j \in \mathbb{N}$. Again, split $\mathbb{N}$ into countably many infinite pairwise disjoint subsets $\mathbb{N}_{i}=\left\{i_{1}<i_{2}<\cdots\right\}, i \in \mathbb{N}$, and define

$$
y_{i}=\sum_{j=1}^{\infty} \xi_{j} e_{i_{j}} \in \mathbb{K}^{\mathbb{N}}
$$

for every $i \in \mathbb{N}$. Since $\left\|y_{i}\right\|_{r}=\|\xi\|_{r}$ for every $r>0$ and $f_{j}(0)=0$ for every $j$, we have that $y_{i} \in C\left(\ell_{p},\left(f_{j}\right)_{j=1}^{\infty}, \Gamma\right)$ for every $i$. As in the proof of Theorem 2.5, for $\lambda=\left(\lambda_{n}\right)_{n=1}^{\infty} \in \mathbb{K}^{\mathbb{N}}$ and $w \in X$, we denote

$$
\lambda \otimes w:=\left(\lambda_{n} w\right)_{n=1}^{\infty} \in X^{\mathbb{N}} .
$$

Define $\tilde{s}=1$ if $p \geq 1$ and $\tilde{s}=p$ if $0<p<1$. For $\left(w_{j}\right)_{j=1}^{\infty} \in \ell_{\tilde{s}}(X)$,

$$
\begin{aligned}
\sum_{j=1}^{\infty}\left\|y_{j} \otimes w_{j}\right\|_{p}^{\tilde{s}} & =\sum_{j=1}^{\infty}\left\|\sum_{k=1}^{\infty} \xi_{k} w_{j} e_{j_{k}}\right\|_{p}^{\tilde{s}}=\sum_{j=1}^{\infty}\left(\sum_{k=1}^{\infty}\left\|\xi_{k} w_{j}\right\|_{X}^{p}\right)^{\tilde{s} / p} \\
& =\sum_{j=1}^{\infty}\left(\sum_{k=1}^{\infty}\left|\xi_{k}\right|^{p} \cdot\left\|w_{j}\right\|_{X}^{p}\right)^{\tilde{s} / p}
\end{aligned}
$$




$$
\begin{aligned}
& =\sum_{j=1}^{\infty}\left\|w_{j}\right\|_{X}^{\tilde{s}} \cdot\left(\sum_{k=1}^{\infty}\left|\xi_{k}\right|^{p}\right)^{\tilde{s} / p} \\
& =\sum_{j=1}^{\infty}\left\|w_{j}\right\|_{X}^{\tilde{s}} \cdot\|\xi\|_{p}^{\tilde{s}}=\|\xi\|_{p}^{\tilde{s}} \cdot\left\|\left(w_{j}\right)_{j=1}^{\infty}\right\|_{\tilde{s}}^{\tilde{s}}<+\infty .
\end{aligned}
$$

Thus, $\sum_{j=1}^{\infty}\left\|y_{j} \otimes w_{j}\right\|_{p}<+\infty$ if $p \geq 1$ and $\sum_{j=1}^{\infty}\left\|y_{j} \otimes w_{j}\right\|_{p}^{p}<+\infty$ if $0<p<1$. It follows that the series $\sum_{j=1}^{\infty} y_{j} \otimes w_{j}$ converges in $\ell_{p}(X)$ and the operator

$$
T: \ell_{\tilde{s}}(X) \longrightarrow \ell_{p}(X), \quad T\left(\left(w_{j}\right)_{j=1}^{\infty}\right)=\sum_{j=1}^{\infty} y_{j} \otimes w_{j},
$$

is well defined. Clearly, $T$ is linear and injective, and thus $\overline{T\left(\ell_{\tilde{S}}(X)\right)}$ is a closed infinite-dimensional subspace of $\ell_{p}(X)$ with

$$
\operatorname{dim} \ell_{p}(X) \geq \operatorname{dim} \overline{T\left(\ell_{\tilde{s}}(X)\right)} \geq \operatorname{dim} T\left(\ell_{\tilde{s}}(X)\right)=\operatorname{dim} \ell_{\tilde{s}}(X)=\operatorname{dim} \ell_{p}(X) ;
$$

hence, $\operatorname{dim} \overline{T\left(\ell_{\tilde{s}}(X)\right)}=\operatorname{dim} \ell_{p}(X)$. Now we just have to show that if $z=$ $\left(z_{n}\right)_{n=1}^{\infty} \in \overline{T\left(\ell_{\tilde{s}}(X)\right)}, z \neq 0$, then $\left(f_{n}\left(\left\|z_{n}\right\|\right)\right)_{n=1}^{\infty} \notin \bigcup_{q \in \Gamma} \ell_{q}$. There are sequences $\left(w_{i}^{(k)}\right)_{i=1}^{\infty} \in \ell_{\tilde{s}}(X), k \in \mathbb{N}$, such that $z=\lim _{k \rightarrow \infty} T\left(\left(w_{i}^{(k)}\right)_{i=1}^{\infty}\right)$ in $\ell_{p}(X)$. Note that, for each $k \in \mathbb{N}$,

$$
\begin{aligned}
T\left(\left(w_{i}^{(k)}\right)_{i=1}^{\infty}\right) & =\sum_{i=1}^{\infty} y_{i} \otimes w_{i}^{(k)} \\
& =\sum_{i=1}^{\infty} \sum_{j=1}^{\infty} \xi_{j} w_{i}^{(k)} e_{i_{j}}
\end{aligned}
$$

Since $z \neq 0$, there is $r \in \mathbb{N}$ such that $z_{r} \neq 0$. Since $\mathbb{N}=\bigcup_{j=1}^{\infty} \mathbb{N}_{j}$, there are (unique) $m, t \in \mathbb{N}$ such that $e_{m_{t}}=e_{r}$. Thus, for each $k \in \mathbb{N}$, the $r$ th coordinate of $T\left(\left(w_{i}^{(k)}\right)_{i=1}^{\infty}\right)$ is $\xi_{t} w_{m}^{(k)}$. Since convergence in $\ell_{p}(X)$ implies coordinatewise convergence, we have

$$
z_{r}=\lim _{k \rightarrow \infty} \xi_{t} w_{m}^{(k)}=\xi_{t} \cdot \lim _{k \rightarrow \infty} w_{m}^{(k)}
$$

and

$$
\lim _{k \rightarrow \infty} w_{m}^{(k)}=\frac{z_{r}}{\xi_{t}} \neq 0
$$

For $j, k \in \mathbb{N}$, the $m_{j}$ th coordinate of $T\left(\left(w_{i}^{(k)}\right)_{i=1}^{\infty}\right)$ is $\xi_{j} w_{m}^{(k)}$. Defining $u_{m}=$ $z_{r} / \xi_{t} \neq 0$, we have

$$
\lim _{k \rightarrow \infty} \xi_{j} w_{m}^{(k)}=\xi_{j} \cdot \lim _{k \rightarrow \infty} w_{m}^{(k)}=\xi_{j} u_{m}
$$

for every $j \in \mathbb{N}$. On the other hand, coordinatewise convergence gives $\lim _{k \rightarrow \infty} \xi_{j} w_{m}^{(k)}=z_{m_{j}}$, so $z_{m_{j}}=\xi_{j} u_{m}$ for each $j \in \mathbb{N}$. By construction we have $m_{j} \leq j$ for every $j$. Let $C$ and $\varepsilon$ be as in Definition 3.1(b). Since $\xi \in \ell_{p}$, there is 
$N \in \mathbb{N}$ such that $\left|\xi_{n}\right|<\varepsilon$ for $n \geq N$. Finally,

$$
\begin{aligned}
\sum_{j=N}^{\infty}\left|f_{m_{j}}\left(\left\|z_{m_{j}}\right\|\right)\right|^{q} & \geq \sum_{j=N}^{\infty}\left[K\left(\left\|u_{m}\right\|\right)\right]^{q} \cdot\left|f_{m_{j}}\left(\left|\xi_{j}\right|\right)\right|^{q} \\
& \geq C \cdot\left[K\left(\left\|u_{m}\right\|\right)\right]^{q} \cdot \sum_{j=N}^{\infty}\left|f_{j}\left(\left|\xi_{j}\right|\right)\right|^{q} \\
& =C \cdot\left[K\left(\left\|u_{m}\right\|\right)\right]^{q} \cdot \sum_{j=N}^{\infty}\left|f_{j}\left(\frac{\xi_{j}}{\xi_{j}}\left|\xi_{j}\right|\right)\right|^{q} \\
& \geq C \cdot\left[K\left(\left\|u_{m}\right\|\right)\right]^{q} \cdot \sum_{j=N}^{\infty}\left[K\left(\frac{\left|\xi_{j}\right|}{\xi_{j}}\right)\right]^{q} \cdot\left|f_{j}\left(\xi_{j}\right)\right|^{q} \\
& \geq C \cdot\left[K\left(\left\|u_{m}\right\|\right)\right]^{q} \cdot\left[\inf _{|\alpha|=1} K(\alpha)\right]^{q} \cdot \sum_{j=N}^{\infty}\left|f_{j}\left(\xi_{j}\right)\right|^{q} \\
& =C \cdot\left[K\left(\left\|u_{m}\right\|\right)\right]^{q} \cdot\left[\inf _{|\alpha|=1} K(\alpha)\right]^{q} \cdot\left\|\left(f_{j}\left(\xi_{j}\right)\right)_{j=N}^{\infty}\right\|_{q}^{q}=+\infty
\end{aligned}
$$

for all $0<q<p$, proving that $\left(f_{n}\left(\left\|z_{n}\right\|\right)\right)_{n=1}^{\infty} \notin \bigcup_{q \in \Gamma} \ell_{q}$.

Let us give a concrete application of Theorem 3.2. In what follows, $\Lambda$ shall be either $[1,+\infty)$ or $(0,1]$. Given a bounded sequence $\left(p_{j}\right)_{j=1} \subseteq \Lambda$ and a Banach space $X$, by $\ell\left(\left(p_{j}\right)_{j=1}^{\infty}, X\right)$ we denote the Nakano space of $X$-valued sequences, that is,

$$
\ell\left(\left(p_{j}\right)_{j=1}^{\infty}, X\right)=\left\{\left(x_{j}\right)_{j=1}^{\infty} \in X^{\mathbb{N}}: \sum_{j=1}^{\infty}\left\|x_{j}\right\|^{p_{j}}<+\infty\right\} .
$$

As usual, when $X=\mathbb{K}$, we simply write $\ell\left(\left(p_{j}\right)_{j=1}^{\infty}\right)$. This space of scalar-valued Nakano sequences was introduced in [22] for $\Lambda=[1,+\infty)$ and has been explored by many authors since then (see, e.g., [18] and references therein). For the case $\Lambda=(0,1]$, see [24]. For vector-valued Nakano sequence spaces, we refer to [7]. The referee kindly pointed out to us that spaceability in scalar-valued Nakano sequence spaces has been recently treated in [23]. As far as we know, (maximal) spaceability involving vector-valued Nakano sequence spaces has never been investigated. In this direction we have the following:

Corollary 3.3. Let $\left(p_{j}\right)_{j=1} \subseteq \Lambda$ be a monotonically nondecreasing bounded sequence, and $p>0$. If $\ell_{p}-\ell\left(\left(p_{j}\right)_{j=1}^{\infty}\right)$ is nonempty, then $\ell_{p}(X)-\ell\left(\left(p_{j}\right)_{j=1}^{\infty}, X\right)$ is maximal spaceable, regardless of the infinite-dimensional Banach space $X$.

Proof. For $j \in \mathbb{N}$, consider the function

$$
f_{j}: \mathbb{K} \longrightarrow \mathbb{K}, \quad f_{j}(\xi)=|\xi|^{p_{j}} .
$$


It is clear that $f_{j}(0)=0$. Let $L \geq 1$ be such that $p_{j} \leq L$ for every $j$. For $\alpha \in \mathbb{K}$, $\alpha \neq 0$, choosing

$$
K(\alpha)= \begin{cases}\min \left\{|\alpha|,|\alpha|^{L}\right\} & \text { if } \Lambda=[1,+\infty), \\ \min \left\{|\alpha|,|\alpha|^{p_{1}}\right\} & \text { if } \Lambda=(0,1],\end{cases}
$$

condition (a) of Definition 3.1 is fulfilled. From the monotonicity of the sequence $\left(p_{j}\right)_{j=1}^{\infty}$, condition (b) holds for $C=\varepsilon=1$. Letting $\Gamma=\{1\}$, we have $C\left(\ell_{p},\left(f_{j}\right)_{j=1}^{\infty}, \Gamma\right)=\ell_{p}-\ell\left(\left(p_{j}\right)_{j=1}^{\infty}\right)$ and $C\left(\ell_{p}(X),\left(f_{j} \circ\|\cdot\|\right)_{j=1}^{\infty}, \Gamma\right)=\ell_{p}(X)-$ $\ell\left(\left(p_{j}\right)_{j=1}^{\infty}, X\right)$, so the result follows from Theorem 3.2.

Of course, the most interesting case occurs when $p_{j} \longrightarrow p$. We can find in the literature several situations where $\ell_{p}-\ell\left(\left(p_{j}\right)_{j=1}^{\infty}\right) \neq \emptyset$ with $\left(p_{j}\right)_{j=1}^{\infty}$ being monotonically nondecreasing and $p_{j} \longrightarrow p$ (hence bounded). For example, in the case $\Lambda=[1,+\infty)$, take $p>1$ and a monotonically nondecreasing sequence $\left(a_{j}\right)_{j=1}^{\infty}$ of real numbers such that $a_{j} \longrightarrow+\infty$ and $\sum_{j=1}^{\infty} C^{a_{j}}=+\infty$ for every $0<C<1$. A concrete example of such a sequence is $a_{j}=1+\log (\log (j+4))$ (see [22, p. 512]). Putting $p_{j}=p-1 / a_{j}$, we obtain a monotonically increasing sequence converging to $p$ such that $\ell_{p}-\ell\left(\left(p_{j}\right)_{j=1}^{\infty}\right) \neq \emptyset$ (cf. [7, Cor. 2.1]). For the case $\Lambda=(0,1]$ and $p=1$, see $[24$, Thm. 3].

AcKnowledgments. The authors thank L. Bernal-González for his helpful suggestions and the referee for his/her careful reading of the paper and for drawing our attention to reference [23].

\section{References}

[1] W. Arendt and R. Nittka, Equivalent complete norms and positivity, Arch. Math. (Basel) 92 (2009), 414-427.

[2] R. M. Aron, D. García, and M. Maestre, Linearity in non-linear problems, Rev. R. Acad. Cienc. Exactas Fís. Nat. Ser. A Math. RACSAM 95 (2001), no. 1, 7-12.

[3] R. M. Aron, V. I. Gurariy, and J. B. Seoane-Sepúlveda, Lineability and spaceability of sets of functions on $\mathbb{R}$, Proc. Amer. Math. Soc. 133 (2005), 795-803.

[4] C. S. Barroso, G. Botelho, V. V. Fávaro, and D. Pellegrino, Lineability and spaceability for the weak form of Peano's theorem and vector-valued sequence spaces, Proc. Amer. Math. Soc. 141 (2013), 1913-1923.

[5] L. Bernal-González and M. Ordoñez Cabrera, Lineability criteria, with applications, J. Funct. Anal. 266 (2014), 3997-4025.

[6] L. Bernal-González, D. Pellegrino, and J. B. Seoane-Sepúlveda, Linear subsets of nonlinear sets in topological vector spaces, Bull. Amer. Math. Soc. (N.S.) 51 (2014), 71-130.

[7] O. Blasco and P. Gregori, Type and cotype in vector-valued Nakano sequence spaces, J. Math. Anal. Appl. 264 (2001), 657-672.

[8] G. Botelho, D. Cariello, D. Pellegrino, and V. V. Fávaro, Maximal spaceability in sequence spaces, Linear Algebra Appl. 437 (2012), 2978-2985.

[9] G. Botelho, D. Diniz, V. V. Fávaro, and D. Pellegrino, Spaceability in Banach and quasi-Banach sequence spaces, Linear Algebra Appl. 434 (2011), 1255-1260. 
[10] G. Botelho, D. Pellegrino, and P. Rueda, A nonlinear Pietsch domination theorem, Monatsh. Math. 158 (2009), 247-257.

[11] _ Dominated polynomials on infinite dimensional spaces, Proc. Amer. Math. Soc. 138 (2010), 209-216.

[12] , A unified Pietsch domination theorem, J. Math. Anal. Appl. 365 (2010), 269-276.

[13] D. Cariello and J. B. Seoane-Sepúlveda, Basic sequences and spaceability in $\ell_{p}$ spaces, J. Funct. Anal. 266 (2014), 3797-3814.

[14] V. P. Fonf, V. I. Gurariy, and M. I. Kadets, An infinite dimensional subspace of $C[0,1]$ consisting of nowhere differentiable functions, C. R. Acad. Bulgare Sci. 52 (1999), no. $11-12,13-16$.

[15] V. P. Fonf and C. Zanco, Almost overcomplete and almost overtotal sequences in Banach spaces, J. Math. Anal. Appl. 420 (2014), 94-101.

[16] V. I. Gurariy, Linear spaces composed of everywhere nondifferentiable functions, C. R. Acad. Bulgare Sci. 44 (1991), no. 5, 13-16 (Russian).

[17] V. I. Gurariy and L. Quarta, On lineability of sets of continuous functions, J. Math. Anal. Appl. 294 (2004), no. 1, 62-72.

[18] A. Kamińska and H. J. Lee, Banach-Saks properties of Musielak-Orlicz and Nakano sequence spaces, Proc. Amer. Math. Soc. 142 (2014), 547-558.

[19] D. Kitson and R. Timoney, Operator ranges and spaceability, J. Math. Anal. Appl. 378 (2011), no. 2, 680-686.

[20] M. C. Matos, Nonlinear absolutely summing mappings, Math. Nachr. 258 (2003), 71-89.

[21] R. E. Megginson, An introduction to Banach space theory, Springer, New York, 1998.

[22] H. Nakano, Modulared sequence spaces, Proc. Japan Acad. 27 (1951), 508-512.

[23] C. Ruiz and V. M. Sánchez, Nonlinear subsets of function spaces and spaceability, Linear Algebra Appl. 463 (2014), 56-67.

[24] S. Simons, The sequence spaces $l\left(p_{v}\right)$ and $m\left(p_{v}\right)$, Proc. Lond. Math. Soc. (3) 3 (1965), 422-436.

G. Botelho

Faculdade de Matemática

Universidade Federal de Uberlândia

38.400-902, Uberlândia

Brazil

botelho@ufu.br
V. V. Fávaro

Faculdade de Matemática

Universidade Federal de Uberlândia

38.400-902, Uberlândia

Brazil 